\title{
INFINITE DIMENSIONAL JORDAN OPERATORS AND STURM-LIOUVILLE CONJUGATE POINT THEORY ${ }^{(1)}$
}

\author{
BY
}

\section{J. WILLIAM HELTON}

ABSTRACT. This article concerns two simple types of bounded operators with real spectrum on a Hilbert space $H$. The purpose of this note is to suggest an abstract algebraic characterization for these operators and to point out a rather unexpected connection between such algebraic considerations and the classical theory of ordinary differential equations. Now some definitions.

A Jordan operator has the form $S+N$ where $S$ is selfadjoint, $N^{2}=0$, and $S$ commutes with $N$. A sub-Jordan operator is the restriction of a Jordan operator $J$ to an invariant subspace of $J$. A coadjoint operator $T$ satisfies $e^{-i s T^{*}} e^{i s T}$ $=I+A_{1} s+A{ }_{2}{ }^{2}$ for some operators $A_{1}$ and $A_{2}$ or equivalently $T^{* 3}-3 T^{* 2} T+$ $3 T^{*} T^{2}-T^{3}=0$.

The main results are

Theorem A. An operator $T$ is Jordan if and only if both $T$ and $T^{*}$ are coadjoint.

Theorem B. If $T$ is coadjoint, if $T$ has a cyclic vector, and if $\sigma(T)=[a, b]$, then $T$ is unitarily equivalent to 'multiplication by $x^{\prime}$ on a weighted Sobolev space of order 1 which is supported on $[a, b]$.

Theorem C. If $T$ is coadjoint and satisfies additional technical assumptions, then $T$ is a sub-Jordan operator.

Let us discuss Theorem C. Its converse, every sub-Jordan operator is coadjoint, is easy to prove. The proof of Theorem C consists of using Theorem B to reduce Theorem $C$ to a question about ordinary differential equations which can be solved by an exacting application of the Jacobi conjugate point theorem for Sturm-Liouville operators. The author suspects that Theorem $C$ is itself related to the conjugate point theorem.

Introduction. This article concerns the two simplest types of bounded operators with real spectrum on a Hilbert space $H$. The purpose of this article is to suggest an abstract algebraic characterization for the se operators and to point out a rather unexpected connection between such algebraic considerations and the classical theory of ordinary differential equations. In particular, our Theorem II which gives an algebraic characterization of certain sub-Jordan operators (defined below) seems very closely related to the classical theorem asserting that a SturmLiouville operator defined on the interval $[a, b]$ is positive definite if and only if there are no points conjugate to $a$ in the interval. One appealing thing is that

Received by the editors August 2, 1971.

AMS 1969 subject classifications. Primary 4730, 4740, 3442; Secondary 4760.

Key words and phrases. Multipliers on a Sobolev space, nonselfadjoint spectral representations, generalized spectral operators, Jordan operators on a Hilbert space, SturmLiouville conjugate point theory.

(1) This research was performed while the author was partially supported by NSF Grant GP-19587. 
almost every idea presented here has a natural generalization worthy of investigation. This is discussed at the end of the article.

The two types of operators considered here are: Jordan operators (order $k$ )operators of the form $S+N$ where $S$ is selfadjoint, $S$ commutes with $N$, and $N^{k}$ $=0 ;$ sub-Jordan operators (order $k$ )-operators which are unitarily equivalent to the restriction of a Jordan operator $J$ to an invariant subspace of $J$.

A natural algebraic condition on a bounded operator $T$ which generalizes the selfadjointness condition is

POL $n$

$$
e^{-i s T^{*}} e^{i s T}=\sum_{k=0}^{n} A_{k} s^{k} .
$$

This is equivalent to $\left(d^{n+1} / d s^{n+1}\right) e^{-i s T^{*}} e^{i s T}=0$ which is in turn equivalent to $C_{T}^{n+1}(I)=0$, where $C_{T}: \mathfrak{L}(H) \rightarrow \mathscr{L}(H)$ is defined by $C_{T}(A)=T^{*} A-A T$ and $C_{T}^{k}$ denotes the composition of the map $C_{T}$ with itself $k$ timess. For example, $T$ satisfies POL 2 if and only if $T^{* 3}-3 T^{* 2} T+3 T^{*} T^{2}-T^{3}=0$. An operator $T$ which satisfies POL $n$ will be called coadjoint (order $n$ ). Note that if $T$ is coadjoint, then $T^{*}$ is not necessarily coadjoint. It is easy to check that every coadjoint operator has real spectrum. This paper concerns only Jordan operators of order 2 and henceforth only these will be called Jordan operators. One major result of this paper $(\$ 1)$ is

Theorem I. An operator $T$ is Jordan (order 2) if and only if botb $T$ and $T^{*}$ are coadjoint (order 2).

Next we turn to sub-Jordan operators. It is obvious that any sub-Jordan operator satisfies the POL condition of the appropriate order and it seems natural to ask

Question. Is any coadjoint operator a sub-Jordan operator? The bulk of this paper is devoted to proving that under strong additional assumptions the answer to this question is yes. I am fairly certain that these additional assumptions can be relaxed greatly (see $\$ 4$ ).

In order to state these additional assumptions, we need some more background. Henceforth assume that $T$ satisfies POL 2. It is clear from POL 2 that $e^{-i s T^{*}} e^{i s T} / s^{2} \rightarrow A_{2}$. This implies two things: first, $A_{2}$ is a nonnegative operator; and second, $e^{-i s T^{*}} A_{2} e^{-i s T}=A_{2}$ for each $s$. If $A_{2}$ has no null vectors, then $[x, y]_{2}=\left[A_{2} x, y\right]$ is a positive definite bilinear form on $H([$,$] denotes the$ inner product on $H$ ) and can be completed to a Hilbert space $H_{2}$ which contains $H$ as a dense subspace. The relation $e^{i s T^{*}} A_{2} e^{-i s T}=A_{2}$ is equivalent to the statement that $e^{i s T}$ is a one parameter unitary group on $H_{2}$. The infinitesimal generator $T$ of this group is selfadjoint and consequently can be thoroughly studied as an operator on $\mathrm{H}_{2}$ by using its spectral resolution 
which we write $T=\int \lambda d E_{\lambda}$. Let $l$ stand for Lebesgue measure. In our main theorem the following will be assumed.

Technical Assumption. There are positive numbers $M$ and $m$ so that for any Borel subset $S$ of $[a, b] m l(S) \leq\left[A_{2} E(S) \psi_{0}, \psi_{0}\right] \leq M l(S)$ where $\psi_{0}$ is the cyclic vector for $T$ mentioned in Theorem II.

Fortunately, this condition is invariant under the right operations, namely, change of cyclic vector. Now we state the main theorem of this article $\left(\$_{3}\right)$.

Theorem II. If $T$ is a coadjoint operator (order 2), if $T$ bas a cyclic vector $\psi_{0}$, if spectrum $T=[a, b]$, and if the Technical Assumption bolds, then $T$ is $a$ sub-Jordan operator (order 2).

One of the tools in proving Theorem II is a representation for coadjoint operators as 'multiplication by $x$ ' on a sort of Sobolev space (see [H]). It is of interest in its own right. In order to state the representation theorem precisely we need some notation.

Let $\mu=\left\langle\mu_{i j}\right\rangle$ be a $(M+1) \times(M+1)$ matrix of finite Borel measures on the interval $[a, b]$ and define a bilinear form $(,)_{\mu}$ on $C^{\infty}[a, b]$ by

$$
(f, g)_{\mu}=\sum_{i, j=0}^{M} \int_{a}^{b} f^{(i)} g^{(j)} d \mu_{i j}
$$

where $b^{(j)}(x)=d^{j} b(x) / d x^{j}$. If $(,)_{\mu}$ is positive definite, we let $H(\mu)$ stand for the completion of $C^{\infty}[a, b]$ in $\|f\|_{\mu}=\sqrt{(f, f)_{\mu}}$. We will say that $H(\mu)$ has order M. One representation theorem in this paper is

Theorem 0. If $T$ is coadjoint (order 2), if $T$ bas a cyclic vector, and if $T$ bas spectrum $[a, b]$, then there is a space $H(\mu)$ of order 1 and a unitary map $U$ : $H \rightarrow H(\mu)$ such that $U T U^{-1}$ is multiplication by $x$ on $H(\mu)$.

This theorem doubtlessly generalizes from $n=2$ to arbitrary $n$ and a straightforward but time consuming reworking of the proof of Theorem 0 should give such a result (see Remark 2.1). Theorem 0 can also be adapted to the case where $T$ has finite multiplicity. A theorem to that effect is proved in $\$ 2$. It is Theorem $0^{\prime}$.

After a few definitions, we shall discuss the proof of Theorem II. Let $C^{n}\left[a, b, \mathbf{C}^{k}\right]$ denote the space of $n$ times continuously differentiable $k$-vector valued functions on $[a, b]$. We shall call an $(n+1) \times(n+1)$ matrix $\mu=\left\langle\mu_{i j}\right\rangle$ of Borel measures positive (positive definite) if and only if the bilinear form

$$
\left\{\left(\begin{array}{c}
f_{0} \\
\vdots \\
f_{n}
\end{array}\right),\left(\begin{array}{c}
g_{0} \\
\vdots \\
g_{n}
\end{array}\right)\right\}_{\mu}=\sum_{i, j=0}^{n} \int_{a}^{b} f_{i} \bar{g}_{j} d \mu_{i j}
$$


is positive (positive definite). If $\mu$ is positive definite then the closure of $C^{0}\left[a, b, \mathbf{C}^{n+1}\right]$ in $\{,\}_{\boldsymbol{\mu}}$ is a Hilbert space which we call $\mathcal{H}(\boldsymbol{\mu})$.

Now suppose that $T$ satisfies the hypothesis of Theorem II. We may take the operator $T$ to be 'multiplication by $x$ ' on $H(\mu)$ for some $2 \times 2$ matrix of measures $\boldsymbol{\mu}=\left\langle\mu_{i j}\right\rangle$. Note that the matrix $\boldsymbol{\mu}$ is not uniquely determined, since one can alter the right side of equation (I.1) with integration by parts without changing its left side. Suppose that one can choose $\mu$ to be positive definite. Let $R$ denote the functions in $\mathcal{H}(\mu)$ of the form $\left(\begin{array}{l}f \\ f\end{array}\right)$. Clearly the map $\tau: H(\mu) \rightarrow R$ given by $\tau f \rightarrow$ $\left(\begin{array}{l}f \\ f\end{array}\right)$ is an isometry of $H(\mu)$ onto $R$. Moreover, the map $f \rightarrow x f$ on $H(\mu)$ induces (under $\tau$ ) the map $\widetilde{T}$ on $R$ given by

$$
\left(\begin{array}{l}
f \\
f^{\prime}
\end{array}\right) \rightarrow\left(\begin{array}{ll}
x & 0 \\
1 & x
\end{array}\right)\left(\begin{array}{l}
f \\
f^{\prime}
\end{array}\right) .
$$

The operator $J$ on $\mathcal{H}(\boldsymbol{\mu})$ given by

$$
J\left(\begin{array}{l}
f_{0} \\
f_{1}
\end{array}\right)=\left(\begin{array}{ll}
x & 0 \\
1 & x
\end{array}\right)\left(\begin{array}{l}
f_{0} \\
f_{1}
\end{array}\right)
$$

is clearly Jordan and $\widetilde{T}$ is $J$ restricted to $R$. Thus the original Hilbert space $H$ is unitarily equivalent to $R$ and the operator $T$ on $H$ under this unitary map goes into $\tilde{T}$ which is $J$ restricted to $R$. Thus Theorem II has been reduced to proving that the matrix $\mu$ can be chosen to be positive definite.

This in fact will be the case if one makes the Technical Assumption. $\$ 3$ is devoted to proving that. The Technical Assumption says precisely that the "highest order" measure $\mu_{22}$ is "equivalent" to Lebesgue measure, i.e. $\mu_{22}=\widetilde{P}(x) d x$ where $m \leq \widetilde{P}(x) \leq M$. The proper inequalities and a change of cyclic vector allow one to work with an inner product of the form

$$
(f, g)_{\Lambda}=\int_{a}^{b} f \bar{g} d \nu_{00}+\int_{a}^{b} f^{\prime} \bar{g}^{\prime} P(x) d x .
$$

One can use a modification of the conjugate point theorem for Sturm-Liouville operators to find a positive definite matrix ${ }^{\circ} \mathcal{}$ of measures such that $(f, g)_{\boldsymbol{\Lambda}}=(f, g)_{\boldsymbol{\Lambda}}$ for all $f, g$ in $C^{1}[a, b, C]$.

Henceforth, in this paper the spectrum of $T$ will always be taken to be $[0,1]$.

A survey of the results and methods of this paper appears in $\left[\mathrm{H}_{2}\right] . \S_{1}$ is the proof of Theorem I. $\$ 2$ is the proof of Theorem 0 and Theorem $0^{\prime}$. $\$ 3$ gives the proof of Theorem II. $\$ 4$ suggests ways of extending Theorems $0, \mathrm{I}$, and II and also describes a geometric approach to the study of coadjoint operators.

1. Jordan operators. The purpose of this section is to prove Theorem I which 
says that if

$$
e^{-i s T^{*}} e^{i s T}=I+s A_{1}+s^{2} A_{2}
$$

and

$$
e^{-i s T} e^{i s T^{*}}=I+s B_{1}+s^{2} B_{2},
$$

then $T$ is a Jordan operator. The equations (1.1) are equivalent to the equations $C_{T}^{3}(I)=C_{T^{*}}^{3}(I)=0$ which when written out in full are

$$
\begin{aligned}
T^{* 3}-3 T^{* 2} T+T^{*} T^{2}-T^{3} & =0, \\
-T^{3}+3 T^{2} T^{*}-3 T T^{* 2}+T^{* 3} & =0 .
\end{aligned}
$$

These equations imply

$$
-T^{* 2} T+T^{*} T^{2}=T^{2} T^{*}-T T^{* 2} .
$$

The main tools of the proof are the following six identities

$$
\begin{aligned}
A_{1} A_{2} & =B_{2} A_{1}, \quad A_{2} A_{1}=A_{1} B_{2}, \\
A_{2} B_{2} & =B_{2} A_{2}=0, \\
A_{1}^{2} & =A_{2}+A_{3} .
\end{aligned}
$$

An immediate consequence of $(1.3)$ is

$$
A_{1}^{2} A_{2}=A_{2} A_{1}^{2}, \quad A_{1}^{2} B_{2}=B_{2} A_{1}^{2} .
$$

Now we prove the identities.

First note that one can expand the exponentials in (1.1) to get

$$
A_{1}=-B_{1}=i\left(T-T^{*}\right)=i C_{T}(I)
$$

and

$$
A_{2}=1 / 2\left[-T^{* 2}+2 T^{*} T-T^{2}\right]=1 / 2 C_{T}^{2}(I)
$$

$$
B_{2}=1 / 2\left[-T^{2}-2 T T^{*}-T^{* 2}\right\rceil=1 / 2 C_{T^{*}}^{2}(I) .
$$

One can use this to compute

$$
\begin{aligned}
& (2 / i) A_{1} A_{2}=-T T^{* 2}+2 T T^{*} T-T^{3}+T^{* 3}-2 T^{* 2} T+T^{*} T^{2}, \\
& (2 / i) B_{2} A_{1}=-T^{* 2} T+2 T T^{*} T-T^{3}+T^{* 3}-2 T T^{* 2}+T^{2} T^{*} .
\end{aligned}
$$

Equation (1.2c) implies that the two right-hand expressions are equal. This proves the first part of identity (1.3) and the second part is proved similarly. Next, observe that equations (1.7) give us 


$$
A_{1}^{2}=-\left[T^{2}-T^{*} T-T T^{*}+T^{* 2}\right]=A_{2}+B_{2}
$$

which is identity (1.5). The identity (1.4) is proved a bit differently. Consider

$$
\left[e^{-i s T^{*}} e^{i s T} / s^{2}\right]\left[e^{-i s T} e^{i s T^{*}} / s^{2}\right]=I / s^{4} .
$$

As $s \rightarrow \infty$ the left-hand side converges to $A_{2} B_{2}$ while the right-hand side converges to zero. Thus $A_{2} B_{2}=0$. The fact that $B_{2} A_{2}=0$ is proved similarly.

The next step is to write down explicitly an order two nilpotent operator $N$ such that $C_{N}^{j}(I)=C_{T}^{j}(I)$ and $C_{N}^{j}{ }^{*}(I)=C_{T}^{j}{ }^{*}(I)$ for every $j$. The appropriate operator is the linear extension of

$$
N x= \begin{cases}-i B_{2} A_{1}^{-1} x & \text { if } x \in \text { Range } A_{1}^{2}, \\ 0 & \text { if } x \perp \text { Range } A_{1}^{2} .\end{cases}
$$

We must verify that $N$ is bounded and that it has the other desired properties.

To prove that $N$ is bounded observe that

$$
\left\|B_{2} y\right\|^{2} \leq\left\|A_{2} y\right\|_{0}^{2}+\left\|B_{2} y\right\|^{2}+\left(B_{2} y, A_{2} y\right)+\left(A_{2} y, B_{2} y\right)
$$

since $A_{2}^{*} B_{2}=A_{2} B_{2}=0=B_{2}^{*} A_{2}$ by (1.4). Thus

$$
\left\|B_{2} y\right\|^{2} \leq\left\|A_{2} y+B_{2} y\right\|^{2}=\left\|B_{1}^{2} y\right\|^{2} \leq\left\|B_{1}\right\|^{2}\left\|B_{1} y\right\|^{2}
$$

by identity (1.5). Since $A_{1}=-B_{1}$, this inequality is equivalent to $N$ being a bounded operator which is bounded by $\left\|B_{1}\right\|$.

In what follows license is taken with the invertibility of $A_{1}$, however, one can keep careful track of the null space of $A_{1}$ and make simple estimates like the one above to insure that the following argument is legitimate. The identities (1.3) and (1.4) imply that

$$
N^{2}=-B_{2} A_{1}^{-1} B_{2} A_{1}^{-1}=-B_{2} A_{2} A_{1}^{-2}=0 .
$$

The identities (1.3) and (1.5) imply that

$$
N-N^{*}=-i B_{2} A_{1}^{-1}-i A_{1}^{-1} B_{2}=-i\left[B_{2}+A_{2}\right] A_{1}^{-1}=-i A_{1}=C_{T}(I) .
$$

The identity (1.3) implies that

$$
1 / 2 C_{N}^{2}(I)=N^{*} N=A_{1}^{-1} B_{2} B_{2} A_{1}^{-1}=A_{2}^{2} A_{1}^{-2} .
$$

However, (1.5) and (1.4) imply that

$$
B_{2}^{2} A_{1}^{-2}+A_{2}^{2} A_{1}^{-2}=A_{1}^{2}=B_{2}+A_{2} .
$$

Each side of the equation is the sum of two selfadjoint operators with orthogonal 
range (see (1.4) and (1.6)); moreover, range $B_{2}^{2} A_{1}^{-2} \subset$ closure range $B_{2}$ and likewise for $A_{2}$. Thus $1 / 2 C_{N}^{2}(I)=A_{2}^{2} A_{1}^{-2}=A_{2}=1 / 2 C_{T}^{2}(I)$. The fact that $C_{N}^{2}(I)=$ $C_{T^{*}}^{2}(I)$ follows from the same type of argument and since all higher commutants with $T, T^{*}, N$ and $N^{*}$ are zero, they are certainly equal. Thus $N$ has the desired properties.

The final step in our proof consists of demonstrating that $T-N=S$ is selfadjoint and that it commutes with $N$. It is easy to see that $S$ is selfadjoint, since

$$
S-S^{*}=T-T^{*}-\left[N-N^{*}\right]=C_{T}(I)-C_{N}(I)=0 .
$$

Now we begin the proof that $S N=N S$. The equality $C_{T}^{2}(I)=C_{N}^{2}(I)$ with a bit of computation yields

$$
S\left(N^{*}-N\right)=\left(N^{*}-N\right) S .
$$

The equality $C_{T}^{3}(I)=0$ with a bit of computation yields

$$
N S^{2}-N^{*} S^{2}-S^{2} N-N S N+3 N^{*} N S-3 S N^{*} N+S^{2} N^{*}+N^{*} S N^{*}=0 .
$$

Rearrange terms in this expression, and use the equality $S^{2}\left(N^{*}-N\right)=\left(N^{*}-N\right) S^{2}$ which follows from (1.8a) to get

$$
0=S^{2}\left(N^{*}-N\right)-\left(N^{*}-N\right) S^{2}=N S N-N^{*} S N^{*}+3 S N^{*} N-3 N^{*} N S .
$$

The right side of this equation factors to give

$$
0=\left(N S+S N^{*}\right) N-N^{*}\left(S N^{*}+N S\right)+2 S N^{*} N-2 N^{*} N S .
$$

One can use equation ( $1.8 \mathrm{a}$ ) to see that the first two terms of the equation above cancel each other, and consequently, one gets

$$
S N^{*} N=N^{*} N S \text {. }
$$

It remains for us to show that if $N^{2}=0, S=S^{*}$, and if $N$ and $S$ satisfy equations (1.8) then $S N=N S$. In order to do this we shall write nilpotent operators with $N^{2}=0$ in a special way. Any operator can be written as $N=W A+J B i$ where $A$ and $B$ are positive selfadjoint operators, $W$ and $J$ are selfadjoint operators which have squares equal to the orthogonal projection onto the closures of range $A$ and range $B$, respectively, $W$ commutes with $A$, and $J$ commutes with $B$. The restriction

$$
0=N^{2}=A^{2}-B^{2}+i[W A J B+J B W A]
$$

forces $A$ to equal $B$ and $W J+J W=0$. Equation (1.8a) is equivalent to the equations $S W A=W A S$, which itself implies $S A=A S$, and $S W=W S$. The operator $N^{*} N$ is equal to $2 A^{2}[1+i J W]$ and so equation $(1.8 \mathrm{~b})$ implies that $S J W=J W S$. Therefore, $S J=J S$ and we have proved that $S$ commutes with $N$. 
2. A representation for coadjoint operators. The following theorem is discussed in $\left[\mathrm{H}_{1}\right]$.

Theorem REP. If $T$ is coadjoint of order $n$, if $T$ bas a cyclic vector $\psi_{0}$, and if $\sigma(T)=[0,1]$, then there is a space $H(\mu)$ of some order and a unitary map $U: H \rightarrow H(\mu)$ such that $U T U^{-1}$ is "multiplication by $x$ " on $H(\mu)$.

In this section we shall refine this the orem to obtain Theorem 0 which adds to the above statement that if $T$ is coadjoint of order 2 then $H .(\mu)$ has order 1 . After proving Theorem 0 , we shall extend Theorem 0 to the finite multiplicity case (Theorem $0^{\prime}$ ). For completeness we sketch a proof of the above theorem before going on to Theorem 0 (for details see $\left[\mathrm{H}_{1}\right]$ ).

Proof of theorem. Define

$$
\phi(f)=\frac{1}{\sqrt{2 \pi}} \int_{-\infty}^{\infty} \hat{f}(s) e^{-i s T} d s
$$

for $f \in \mathcal{S}(R)$, the Schwartz space of $C^{\infty}$ functions all of whose derivatives decrease faster than any polynomial at infinity (this is a norm convergent integral because $T$ satisfies POL $n$ ). The map $\phi$ is a continuous algebraic homomorphism of $\mathcal{S}(R)$ into $\mathcal{L}(H)$. By the Schwartz Nuclear Theorem, there is distribution $b$ on $\mathcal{S}\left(R^{2}\right)$, the Schwartz space on $R^{2}$, such that

$$
b(f(x) \bar{g}(y))=\left[\phi(f) \psi_{0}, \phi(g) \psi_{0}\right]
$$

for each $f_{0} g \in \mathcal{S}$. Here $[$,$] denotes the Hilbert space H$ inner product. The POL $n$ condition is equivalent to

$$
0=\frac{d^{n+1}}{d s^{n+1}} b\left(e^{i s}[x-y] f(x) \bar{g}(y)\right)=b\left([x-y]^{n+1} f(x) \bar{g}(y)\right)
$$

which implies that the distribution $b$ has support on the line $\{(x, x)\}$. Moreover, one can apply the Gelfand representation for the Banach algebra of operators of the form $\phi(f)$, and get that $\Delta=\{(x, x): x \in \sigma(T)=[0,1]\}$ is the support of $b$. The usual representation for distributions (Schwartz Kernel Theorem) allows us to write $b$ as

$$
b(b(x, y))=\sum_{i, j=0}^{\mathrm{H}} \int_{0}^{1} K_{i j}(x, x) \frac{\partial^{i+j}}{\partial x^{i} \partial y^{j}} b(x, x) d x
$$

for some $K_{i j}$. Thus the bilinear form $(f, g)=\left[\phi(f) \psi_{0}, \phi(g) \psi_{0}\right]$ on $C^{\infty}[0,1]$ has the representation

$$
(f, g)=\sum_{i, j=0}^{M} \int_{0}^{1} f^{(i)} \bar{g}^{(j)} d \mu_{i j}
$$


for a matrix $\mu=\left\langle\mu_{i j}\right\rangle$ of measures, and $H(\mu)$ of our theorem is precisely the closure of $C^{\infty}[0,1]$ in $($,$) . Since \phi$ is an algebraic homomorphism, $T$ on $H$ corresponds to "multiplication by $x$ " on $H(\mu)$. Our theorem is proved.

The next long while is spent in proving Theorem 0 , in other words, in proving that if $T$ satisfies POL 2, then $M$ in (2.1) is equal to 1 . In the proof of the above theorem when one is careful with the order of the various distributions which appear, one can prove representation (2.1) with $M=2$. Theorem 0 will thus be a consequence of the following proposition.

Proposition 2.1. If

REP

$$
(f, g)=\sum_{i=0 ; j=0}^{2} \int_{0}^{1} f^{(i)} \bar{g}^{(j)} d \mu_{i j}
$$

is a nonnegative bermitian bilinear form on $C^{2}[0,1]$ with $\mu_{i j}$ finite Borel measures and $\left(d^{3} / d s^{3}\right)\left(e^{i s x} f, e^{i s x} g\right)=0$, then in REP the sum on $i$ and $j$ need only go up to 1 .

Proof. If we put $e^{i s x} f$ and $e^{i s x} g$ into REP for $f$ and $g$ and expand in powers of $s$ we get

$$
\begin{aligned}
\left(e^{i s x} f, e^{i s x} g\right)= & s^{4} \int_{0}^{1} f \bar{g} d \mu_{22}+s^{3}\left\{i \int_{0}^{1} f \bar{g} d \mu_{21}-i \int_{0}^{1} f \bar{g} d \mu_{12}\right\} \\
& +s^{2} B_{2}(f, g)+s B_{1}(f, g)+(f, g)
\end{aligned}
$$

where the $B_{j}(f, g)$ are bilinear forms including only $f, g$ and derivatives of $f$ and g. By hypothesis the coefficients of $s^{4}$ and $s^{3}$ are zero, so $\mu_{22}=0$ and $\mu_{21}=$ $\mu_{12}$. When we compute $B_{2}(f, g)$ and use this information we get

$$
B_{2}(f, g)=\int_{0}^{1} f \bar{g} d \mu_{11}+\int_{0}^{1}(f \bar{g})^{\prime} d \mu_{12}-\int_{0}^{1} f \bar{g} d \mu_{02}-\int_{0}^{1} f \bar{g} d \mu_{20} .
$$

Now our hypothesis implies that $B(f, g)=p(f \bar{g})$ where $p$ is a positive linear functional on $C^{2}[0,1]$. Thus $B(f, g)$ is supremum norm continuous and $\int_{0}^{1}(\bar{g})^{\prime} d \mu_{12}$ is supremum norm continuous. By the Baire-Riesz representation theorem $\int_{0}^{1} b^{\prime} d \mu_{12}=\int_{0}^{1} b d \nu$, which together with the fact that $\mu_{12}=\mu_{21}$ implies that we may take $(f, g)$ to have the representation REP with $\mu_{12}, \mu_{21}$ identically zero and $\mu_{22}$ identically zero (Note: when $($,$) is written this way, the measures \mu_{i j}$ may be different from the $\mu_{i j}$ that we begin with).

The remaining problem is to eliminate $\mu_{02}$ and $\mu_{20}$. Our plan is to prove that $\mu_{20}$ and $\mu_{02}$ each have derivatives which are measures denoted by $\nu_{1}$ and $\nu_{2}$ respectively. Then we can write 


$$
\begin{aligned}
\int_{0}^{1} f^{\prime \prime} \bar{g} d \mu_{20}+\int_{0}^{1} f \bar{g}^{\prime \prime} d \mu_{02}= & -\int_{0}^{1} f^{\prime} \bar{g}^{\prime} d \mu_{20}-\int_{0}^{1} f^{\prime} \bar{g} d \nu_{2}-\int_{0}^{1} f^{\prime} \bar{g}^{\prime} d \mu_{02} \\
& -\int_{0}^{1} f \bar{g}^{\prime} d \nu+\int_{0}^{1} f^{\prime} \bar{g} d \delta_{0}+\int_{0}^{1} f \bar{g}^{\prime} d \delta_{1}
\end{aligned}
$$

where $\delta_{0}$ and $\delta_{1}$ are measures which are concentrated at the end points of $[0,1]$. This means that $(f, g)$ can be written in the form required by our theorem. To prove that $\mu_{20}$ and $\mu_{02}$ are differentiable it suffices to show that they are absolutely continuous with respect to Lebesgue measure $l$ and that $d \mu_{02} / d l$ and $d \mu_{20} / d l$ are of bounded variation (cf. [R, Chapter $\left.12, \S 3\right]$ ). The proof consists of five steps which show that the measures in REP can be chosen in nicer and nicer ways. In each step when we refer to REP we shall assume that the measures appearing in it have all of the nice properties which were gotten for them in the preceding steps.

Step 1. The measure $\mu_{02}-\mu_{20}$ has a derivative which is a measure (i.e. $\int_{0}^{1} f^{\prime} d\left[\mu_{02}-\mu_{20}\right]$ is supremum norm continuous in $f$ ).

Proof. Suppose not. Suppose that $b_{n}$ is a sequence of real valued functions in $C^{2}[0,1]$ such that $\left|b_{n}\right| \leq 1$ and $\left|b_{n}^{\prime}\right| \leq 1$, and that $\left|\int_{0}^{1} b_{n}^{\prime \prime} d\left[\mu_{02}-\mu_{20}\right]\right| \rightarrow \infty$. It follows from REP that

$$
\begin{aligned}
\left(e^{i b}, e^{i b}\right)= & \int_{0}^{1} d \mu_{00}+i \int_{0}^{1} b^{\prime} d \mu_{10}-i \int_{0}^{1} b^{\prime} d \mu_{01}+\int_{0}^{1} b^{\prime 2} d \mu_{11} \\
& +\int_{0}^{1}\left(i b^{\prime \prime}-b^{\prime 2}\right) d \mu_{20}-\int_{0}^{1}\left(i b^{\prime \prime}-b^{\prime 2}\right) d \mu_{02^{\circ}}
\end{aligned}
$$

If we let $b=b_{n}$ and put the second order terms first we get

$$
\left(e^{ \pm i b_{n}}, e^{ \pm i b_{n}}\right)= \pm i \int_{0}^{1} b_{n}^{\prime \prime} d\left[\mu_{02}-\mu_{20}\right]+G\left( \pm b_{n}\right)
$$

where $\left|G\left( \pm b_{n}\right)\right|$ is uniformly bounded by a number $M$. However, for large enough $n$ the first term on the right side of the equation has absolute value bigger than $M$. For such $n$ the right side of the equation can be made either complex or negative by an appropriate choice of sign. This contradicts the fact that the left side must be positive.

Step 2. We may take $\mu_{00}, \mu_{11}, \mu_{02}$ to be real measures and also we may take $\mu_{01}=\bar{\mu}_{10}$.

Proof. We write the right-hand side of REP as the sum of a hermitian and skew-hermitian part

$$
\begin{aligned}
(f, g)= & \frac{1}{2} \int_{0}^{1} f \bar{g} d\left[\mu_{00}+\bar{\mu}_{00}\right]+\frac{1}{2} \int_{0}^{1} f \bar{g}^{\prime} d\left[\mu_{01}+\bar{\mu}_{10}\right]+\frac{1}{2} \int_{0}^{1} f^{\prime} \bar{g} d\left[\mu_{10}+\bar{\mu}_{01}\right] \\
& +\frac{1}{2} \int_{0}^{1} f^{\prime} \bar{g}^{\prime} d\left[\mu_{11}+\bar{\mu}_{11}\right]+\frac{1}{2} \int_{0}^{1} f^{\prime \prime} \bar{g}+f \bar{g}^{\prime \prime} d\left[\mu_{02}+\bar{\mu}_{02}\right] \\
& +\frac{1}{2} \int_{0}^{1} f \bar{g}^{\prime} d\left[\mu_{01}-\bar{\mu}_{10}\right]+\frac{1}{2} \int_{0}^{1} f^{\prime} \bar{g} d\left[\mu_{10}-\bar{\mu}_{01} 1+\frac{1}{2} \int_{0}^{1} f^{\prime \prime} \bar{g}+f \bar{g}^{\prime \prime} d\left[\mu_{02}-\bar{\mu}_{02}\right]\right.
\end{aligned}
$$


Since the left side of the equation is hermitian the last three terms in the equation must be hermitian and skew-hermitian simultaneously. Thus the last three terms in the equation sum to zero and $(f, g)$ can be written simply as the sum of the first five terms on the right side of the equation. The measures $\mu_{00}^{\prime}=$ $1 / 2\left(\mu_{00}+\bar{\mu}_{00}\right)$ etc. used in this new representation for $($,$) have the required prop-$ erties.

step 3. The measure $\mu_{02}$ is absolutely continuous with respect to Lebesgue measure.

Proof. Suppose not. Decompose $\mu_{02}$ into positive and negative parts. Without loss of generality we may suppose that the positive part $\nu$ of $\mu_{02}$ is not absolutely continuous with respect to Lebesgue measure $l$; let $S$ be a set such that $\nu(S) \neq 0$ but $l(S)=0$. There is a sequence $S_{N}^{\prime}$ of open sets containing $S$ such that $\nu\left(S_{N}^{\prime}\right) \rightarrow \nu(S)$ and $l\left(S_{N}^{\prime}\right) \rightarrow 0$. Also, there is a sequence $S_{M}^{\prime \prime}$ of open sets containing the support $P$ of the positive part $\nu$ of $\mu_{02}$ with the property that $\left|\mu_{02}\right|\left(S_{M}^{\prime \prime}\right) \rightarrow\left|\mu_{02}\right|(P)=\nu(P)$. Set $S_{N}=S_{N}^{\prime} \cap S_{N}^{\prime \prime}$. There is a sequence $C_{N}$ of closed sets contained in $S$ such that $\nu\left(C_{N}\right) \rightarrow \nu(P)$. By Urysohn's lemma there is a positive real valued function $b_{N}$ which is identically 1 on $C_{N}$, identically 0 off of $S_{N}$, bounded by 1 on $[0,1]$, and which is continuous on $[0,1]$. Define $b_{N}(x)=\alpha_{N} \int_{0}^{x} \int_{0}^{s} b_{N}(t) d t d s+\gamma$ where $\alpha_{N}$ and $\gamma$ are constants.

The following estimates hold for the sizes of $b_{N}$ and $b_{N}^{\prime}$

$$
\begin{aligned}
& \left|b_{N}^{\prime}(x)\right|=\left|\alpha_{N} \int_{0}^{x} b_{N}(s) d x\right| \leq\left|a_{N}\right| l\left(s_{N}\right), \\
& \left|b_{N}(x)\right|=\left|\int_{0}^{x} b_{N}^{\prime}(s) d s+\gamma\right| \leq\left|\alpha_{N}\right| l\left(s_{N}\right)+|y| .
\end{aligned}
$$

Thus, if we set $a_{N}=1 / l\left(s_{N}\right)$, we get

$$
\left|b_{N}^{\prime}(x)\right| \leq 1, \quad\left|b_{N}(x)\right| \leq 1+|\gamma|
$$

We need another estimate before we can begin. Let $k_{N}=b_{N}-\gamma$; it is a positive function of height less than 1 . We have

$$
\begin{aligned}
\left|\int_{0}^{1} b_{N} k_{N} d \mu_{02}\right| & \leq \int_{0}^{1} b_{N} k_{N} d\left|\mu_{02}\right| \leq \int_{0}^{1} b_{N} d\left|\mu_{02}\right| \\
& \leq \int_{S_{N} \sim C_{N}} b_{N} d\left|\mu_{02}\right|+\nu\left(C_{N}\right), \\
& \left|\int_{0}^{1} b_{N} k_{N} d \mu_{02}\right| \leq \nu\left(C_{N}\right)+\left|\mu_{02}\right|\left(S_{N} \sim C_{N}\right) .
\end{aligned}
$$

Also 


$$
\left|\int_{0}^{1} b_{N} d \mu_{02}-\nu\left(C_{N}\right)\right| \leq \int_{S_{N} \sim C_{N}} b_{N} d\left|\mu_{02}\right| \leq\left|\mu_{02}\right|\left(S_{N} \sim C_{N}\right) .
$$

Next we note that $\left|\mu_{02}\right|\left(S_{N} \sim C_{N}\right) \rightarrow 0$ as $N \rightarrow \infty$. This follows because

$$
\begin{aligned}
\left|\mu_{02}\right|\left(S_{N} \sim C_{N}\right) & =\left|\mu_{02}\right|\left(S_{N} \sim P\right)+\left|\mu_{02}\right|\left(P \sim C_{N}\right) \\
& \leq\left|\mu_{02}\right|\left(S_{N}^{\prime \prime} \sim P\right)+\nu\left(P \sim C_{N}\right)
\end{aligned}
$$

and the terms on the right go to zero by construction. Substitute $b_{N}$ into REP for $f$ and $g$ and use the estimates (2.2), (2.3) to get

$$
\left(b_{N}, b_{N}\right)=2 \int_{0}^{1} b_{N}^{\prime \prime} b_{N} d \mu_{02}+G\left(b_{N}\right)
$$

where $\left|G\left(b_{N}\right)\right|$ is less than or equal to a constant $M_{\gamma}$ which depends only on $\gamma$. Set $b_{N}=k_{N}+\gamma$ and use the fact that $\left(b_{N}, b_{N}\right) \geq 0$ to get

$$
\gamma \int_{0}^{1} b_{N} d \mu_{02} \geq-G\left(b_{N}\right) / 2 \alpha_{N}-\int_{0}^{1} b_{N} k_{N} d \mu_{02}
$$

for each $\gamma$ and N. Estimates (2.4) and (2.5) imply that

$$
(\gamma+1)_{\nu}\left(C_{N}\right) \geq-M_{\gamma} / 2 \alpha_{N}-(2+\gamma)\left|\mu_{02}\right|\left(S_{N} \sim C_{N}\right)
$$

for all $N$ and $\gamma$. Since for fixed $\gamma$ the right side goes to 0 as $N \rightarrow \infty$, this is impossible. Step 3 is now complete. Let $a$ denote the Radon-Nikodym derivative of $\mu_{02}$ with respect to Lebesgue measure; henceforth we shall write $d \mu_{02}=a d x$.

Step 4. $\alpha$ is essentially bounded.

Proof. Suppose not. Define $E_{N} \subset[0,1]$ with $N$ an integer by

$$
E_{N}=\{x \in[0,1]: \alpha(x) \geq N\} .
$$

Without loss of generality we may assume that $l\left(E_{N}\right) \neq 0$ for each $N$. Let $\chi_{N}$ denote the characteristic function of $E_{N}$, i.e. $\chi_{N}(x)=0$ if $x \notin E_{N}, \chi_{N}(x)=1$ if $x \in E_{N}$. Define

$$
b_{N}(x)=\frac{1}{l\left(E_{N}\right)} \int_{0}^{x} \int_{0}^{s} \chi_{N}(t) d t d s+\gamma=K_{N}+\gamma .
$$

Now substitute $b_{N}$ into REP and get

$$
0 \leq\left(b_{N}, b_{N}\right)=\frac{1}{l\left(E_{N}\right)} \int_{0}^{1} \chi_{N}(x) b_{N}(x) \alpha(x) d x+G\left(b_{N}\right)
$$

where $G\left(b_{N}\right)$ is uniformly bounded as $N \rightarrow \infty$. The proof in this part is so similar to the proof in Step 3 that we shall not belabor the estimates. Inequality (2.6) leads to a contradiction, since we may take $\gamma$ to be a negative number with $|\gamma|>$ $1+\left(1 / l\left(E_{N}\right)\right)\left|K_{N}\right|$ and clearly 


$$
\frac{1}{l\left(E_{N}\right)} \int_{E_{N}} \alpha(x) d x \geq \frac{1}{l\left(E_{N}\right)} l\left(E_{N}\right) N \rightarrow \infty .
$$

Step 5. $a(x)$ is a function of essentially bounded variation.

Proof. Recall that the Lebesgue set $A$ of $\alpha$ is a set of $l$-measure 1 such that $p \in A$ if and only if

$$
\lim _{l(C) \rightarrow 0} \int_{C}|\alpha(x)-\alpha(p)| d x=0
$$

where $C$ denotes an arbitrary closed interval containing $p$. We shall let $j_{\epsilon}(x-s)$ be a $C^{\infty}$ reproducing sequence, that is, $j_{\epsilon}(y)=(1 / \epsilon) j(y / \epsilon)$ where $j$ has the properties:

(1) $j \in C^{\infty}, j \geq 0$;

(2) support $j \subset[-1 / 2,1 / 2]$;

(3) $\int_{-1}^{1} j d x=1$;

(4) $j(t)$ is increasing for $t \leq 0$ and decreasing for $t \geq 0$.

A basic fact is that $\int_{0}^{1} j_{\epsilon}(x-p) \alpha(x) d x \rightarrow \alpha(p)$ if $p \in A \cap(0,1)$ (see [D-S, III. 12.10, Theorem 10]).

Suppose that $R_{m}$ is a partition of $(0,1)$ into $2 m$ points in $A$ with the property that $a\left(x_{k}^{m}\right)-a\left(x_{k-1}^{m}\right)>0$ if $k=2,4,6, \ldots, 2 m$. Define

$$
p_{m}=\sum_{k=\text { even }}^{2 m}\left[\alpha\left(x_{k}^{m}\right)-\alpha\left(x_{k-1}^{m}\right)\right] \text {. }
$$

We shall say that $\alpha$ has essentially bounded positive variation if $\sup p_{m}$ over all admissible $R_{m}$ is finite. The negative variation of $\alpha$ is handled similarly. Suppose that $a$ is not of essentially bounded variation. With no loss of generality, we may assume that there is a sequence of $R_{m}$ for which $p_{m} \rightarrow \infty$.

Define $b_{m, \epsilon}$ and $f_{m, \epsilon}$ by

$$
\begin{aligned}
& b_{m, \epsilon}(x)=\sum_{k=\text { even }}^{2 m} j_{\epsilon}\left(x-x_{k}^{m}\right)-j_{\epsilon}\left(x-x_{k-1}^{m}\right), \\
& f_{m, \epsilon}(x)=\int_{0}^{x} \int_{0}^{s} b_{m, \epsilon}(t) d t d s .
\end{aligned}
$$

Note that $\lim _{\epsilon \rightarrow 0} \int_{0}^{1} b_{m, \epsilon} a d x=p_{m}$. Also note that $\left|f_{m, \epsilon}\right|$ and $\left|f_{m, \epsilon}^{\prime}\right|$ are bounded by 1 independently of $m$ and $\epsilon$. Hence, we assume and throughout the rest of this proof we shall assume that $\epsilon<\min \operatorname{dist}\left(x_{i}^{m}, x_{l}^{m}\right)$.

Substitute $f_{m, \epsilon}+\gamma$, where $\gamma$ is a complex number, into REP for both $f$ and $g$ and use the fact that $(f, f) \geq 0$ to obtain

$$
0 \leq 2 \int_{0}^{1} f_{m, \epsilon} b_{m, \epsilon} \alpha d x+2 y \int_{0}^{1} b_{m, \epsilon} \alpha d x+G\left(f_{m, \epsilon}\right)
$$


where $G\left(f_{m, \epsilon}\right)$ is bounded independently of $m$ and $\epsilon$ by a number $M$. Suppose that we can show that there is a number $\Gamma$ such that

$$
\Gamma+\Gamma\left|\int_{0}^{1} b_{m, \epsilon} \alpha d x\right| \geq \int_{0}^{1} f_{m, \epsilon} b_{m, \epsilon} \alpha d x
$$

for all $m$ and $\epsilon_{m}<\delta_{m}$ where $\delta_{m} \rightarrow 0$ as $m \rightarrow \infty$. This implies that $0 \leq$ $2(\Gamma+\gamma) p_{m}+M$ for all $\gamma$ and $p_{m}$. However, we can take $\gamma=-2 \Gamma$ and since $p_{m} \rightarrow \infty$, we have a contradiction.

Now we prove (2.9). Abbreviate $j_{\epsilon}\left(x-x_{i}\right)$ to $j_{\epsilon}(i)$, let $f$ be a function in $C^{\prime}[0,1]$ and consider the following equation:

$$
\begin{aligned}
\int_{0}^{1}\left(j_{\epsilon}(2)\right. & \left.-j_{\epsilon}(1)\right) f a d x \\
= & \int_{0}^{1} j_{\epsilon}(2) f a d x-\int_{0}^{1} j_{\epsilon}(2) \alpha d x \int_{0}^{1} j_{\epsilon}(2) f d x \\
& +\int_{0}^{1} j_{\epsilon}(2) \alpha d x \int_{0}^{1}\left[j_{\epsilon}(2)-j_{\epsilon}(1)\right] f d x-\int_{0}^{1} j_{\epsilon}(1) f a d x \\
& +\int_{0}^{1} j_{\epsilon}(1) \alpha d x \int_{0}^{1} j_{\epsilon}(1) f d x-\int_{0}^{1} j_{\epsilon}(1) f d x \int_{0}^{1}\left[j_{\epsilon}(1)-j_{\epsilon}(2)\right] \alpha d x
\end{aligned}
$$

The following lemma gives us an estimate on the first and second and the fourth and fifth terms on the right-hand side of the equation.

Lemma 2.2. If $x_{0} \in(0,1) \cap A$ and $\nu>0$, then there is a $\delta$ such that

$$
\begin{aligned}
& \left|F_{\epsilon}\left(x_{0}, f\right)\right| \\
& \quad \equiv\left|\int_{0}^{1} \alpha(x) f(x) j_{\epsilon}\left(x-x_{0}\right) d x-\int_{0}^{1} \alpha(x) j_{\epsilon}\left(x-x_{0}\right) d x \int_{0}^{1} f(x) j_{\epsilon}\left(x-x_{0}\right) d x\right|<\nu
\end{aligned}
$$

for all $f$ in the unit ball of $C^{\prime}[0,1]$ and for all $\epsilon<\delta$.

Proof. Suppose that $f \in C^{\prime}[0,1]$. Since $F_{\epsilon}\left(x_{0}, f-f\left(x_{0}\right)\right)=F_{\epsilon}\left(x_{0}, f\right)$, we may assume that $f\left(x_{0}\right)=0$. The last term of $F_{\epsilon}$ can be disregarded because

$$
\left|\int_{0}^{1} f(x) j_{\epsilon}\left(x-x_{0}\right) d x\right| \leq \sup _{\left[x_{0}-\epsilon / 2, x_{0}+\epsilon / 2\right]}|f| \leq(\epsilon / 2) \sup _{[0,1]}\left|f^{\prime}\right|
$$

and because $\int_{0}^{1} \alpha(x) j_{\epsilon}\left(x-x_{0}\right) d x \rightarrow \alpha\left(x_{0}\right)$. To estimate the first term of $F_{\epsilon}\left(x_{0}, f\right)$ we consider

$$
\frac{1}{l(C)} \int_{C}\left|f(x) \alpha(x)-f\left(x_{0}\right) \alpha\left(x_{0}\right)\right| d x
$$

Since $f\left(x_{0}\right)=0$ it has an absolute value dominated by

$$
\frac{1}{l(C)} \int_{C}\left|a(x)-a\left(x_{0}\right)\right| d x \sup _{x \in C}|f(x)|+\frac{1}{l(C)}\left|\alpha\left(x_{0}\right)\right| \int_{C}|f(x)| d x
$$


which, if we take $C=\left[x-\epsilon, x_{0}+\epsilon\right]$, is dominated by

$$
a 2 \epsilon \sup _{[0,1]}\left|f^{\prime}\right|+\left|\alpha\left(x_{0}\right)\right| 2 \epsilon \sup _{[0,1]}|f|,
$$

where $a$ is the supremum of $|\alpha(x)|$. Thus we have

$$
\frac{1}{2 \epsilon} \int_{x_{0}-\epsilon}^{x_{0}+\epsilon}\left|f(x) \alpha(x)-f\left(x_{0}\right) \alpha\left(x_{0}\right)\right| d x \leq \epsilon M\left\{\sup _{[0,1]}\left|f^{\prime}\right|+\sup _{[0,1]}|f|\right\} .
$$

By the usual method, see [D-S, III. 12.10], one can prove that

$$
\left|\int_{0}^{1} \alpha(x) f(x) j_{\epsilon}\left(x-x_{0}\right) d x\right| \leq \sigma(\epsilon)\left\{\sup _{[0,1]}\left|f^{\prime}\right|+\sup _{[0,1]}|f|\right\}
$$

where $\sigma(\epsilon) \rightarrow 0$ as $\epsilon \rightarrow 0$. This completes the proof.

Lemma 2.2 along with the inequalities

$$
\begin{gathered}
\int_{0}^{1}\left[j_{\epsilon}(2)-j_{\epsilon}(1)\right] f d x \leq\left[\left(x_{2}-x_{1}\right)+\epsilon\right] \sup _{[0,1]}\left|f^{\prime}\right|, \\
\int_{0}^{1} j_{\epsilon}(1) f d x \leq \sup _{[0,1]}|f|,
\end{gathered}
$$

when applied to (2.10), imply that for any $\nu>0$, there exists a $\delta>0$ such that

$$
\begin{aligned}
& \left|\int_{0}^{1}\left[j_{\epsilon}(2)-j_{\epsilon}(1)\right] f \alpha d x\right| \\
& \quad \leq \nu+\left|\int_{0}^{1} j_{\epsilon}(2) \alpha d x\left[\left(x_{2}-x_{1}\right)+\nu\right]\right|+\left|\int_{0}^{1}\left[j_{\epsilon}(1)-j_{\epsilon}(2)\right] \alpha d x\right|
\end{aligned}
$$

if $\epsilon<\delta$, if $\epsilon<\nu$, and if $f$ is in the unit ball of $C^{\prime}[0,1]$. Now we apply this estimate to get (2.9)

$$
\begin{aligned}
\left|\int_{0}^{1} b_{m, \epsilon} f_{m, \epsilon} \alpha d x\right|= & \sum_{k=\text { eve n }}^{2 m} \int_{0}^{1}\left[j_{\epsilon}\left(x-x_{k}^{m}\right)-j_{\epsilon}\left(x-x_{k-1}^{m}\right)\right] f_{m, \epsilon} \alpha d x \\
\leq & \sum_{k=\text { even }}^{2 m} \nu\left[1+\int_{0}^{1} j_{\epsilon}\left(x-x_{k}^{m}\right) \alpha d x\right] \\
& +\sum_{k=\text { even }}^{2 m}\left(x_{k}^{m}-x_{k-1}^{m}\right)\left|\int_{0}^{1} j_{\epsilon}\left(x-x_{k}^{m}\right) \alpha d x\right| \\
& +\sum_{k=\text { even }}^{2 m}\left|\int_{0}^{1}\left[j_{\epsilon}\left(x-x_{k}^{m}\right)-j_{\epsilon}\left(x-x_{k-1}^{m}\right)\right] \alpha d x\right|
\end{aligned}
$$

for any $\epsilon$ less than some $\delta_{m}$. Since $\int_{0}^{1} j_{\epsilon}\left(x-x_{k}^{m}\right) \alpha d x \rightarrow a\left(x_{k}^{m}\right)$ for any $x_{k}^{m}$ in $R_{m}$ and since $\alpha\left(x_{k}^{m}\right)-\alpha\left(x_{k-1}^{m}\right)>0$ for $k$ even, a $\delta_{m}$ can be picked with the 
property that

$$
\left|\int_{0}^{1} b_{m, \epsilon} f_{m, \epsilon} \alpha d x\right| \leq 1+\sum_{k=\text { even }}^{2 m}\left|\alpha\left(x_{k}^{m}\right)\right|\left(x_{k}^{m}-x_{k-1}^{m}\right)+\int_{0}^{1} b_{m, \epsilon} \alpha d x
$$

for any $\epsilon<\delta_{m}$. The fact that $\alpha$ is essentially bounded implies that the second term on the right is bounded, say by $\Gamma-1$, from which (2.9) follows. Thus Step 5 is finished and Proposition 2.1 as well as Theorem 0 are proved.

Remark 2.1. It appears (although the author has not checked it) that a more complicated argument along the lines of the preceding one will give:

If $T$ is $n$-power adjoint, then it can be represented as multiplication by $x$ on a generalized Sobolev space $H(\mu)$ of order $n / 2$.

A modification (Steps $3,4,5$ ) in the preceding argument which should give the more general case is this. Take $b$ 's as before but take $b$ 's and f's to be the $n$th integral of the $b$ 's. Instead of substituting $f+\gamma$ into REP, substitute $f+\gamma_{1}+$ $\gamma_{2} x+\cdots+\gamma_{n-2} x^{n-2}$ into REP. Then pick the right $\gamma_{i}$ 's. All of this of course must be combined with an induction argument.

In the last part of this section we shall weaken the requirement in Theorem 0 that $T$ has a cyclic vector. A set $\psi_{0}, \ldots, \psi_{k}$ is called cyclic for $T$ if linear combinations of the vectors $T^{n} \psi_{i}, n=0,1, \ldots, i=1, \ldots, k$, are dense in $H$. Suppose that $T$ has a finite cyclic set $\psi_{0}, \ldots, \psi_{k}$. We shall study the positive (but not necessarily positive definite) form

$$
(F, G)=\left[\phi\left(f_{0}\right) \psi_{0}+\cdots+\phi\left(f_{k}\right) \psi_{k}, \phi\left(f_{0}\right) \psi_{0}+\cdots+\phi\left(f_{k}\right) \psi_{k}\right]
$$

where $F$ and $G$ are vector valued functions in $C^{\infty}\left[R, C^{k}\right]$ with compact support and whose components are $F=\left(f_{0}, \cdots, f_{k}\right)$ and $G=\left(g_{0}, \cdots, g_{k}\right)$.

Let $\mu_{i j}$ be a $k \times k$ matrix of finite Borel measures on $[0,1]$ for $i, j=0,1$, $\cdots, M$. If $F, G \in C^{0}\left[0,1, C^{k}\right]$ define

$$
\int_{0}^{1} d\left\langle\mu_{i j} F, G\right\rangle=\sum_{\alpha, \beta=1}^{k} \int_{0}^{1} f_{a} \bar{g}_{\beta} d \mu_{\alpha \beta}
$$

where $\mu_{\alpha \beta}$ are the entries of $\mu_{i j}$.

Also \langle\rangle will be used to denote the $C^{k}$ inner product. Define a map $U$ : $C^{\infty}\left[0,1, C^{k}\right] \rightarrow H$ by

$$
U(F)=\phi\left(f_{0}\right) \psi_{1}+\cdots+\phi\left(f_{k}\right) .
$$

Clearly, $(F, G)=[U(F), U(G)]$ and it can be checked that $U(x F)=T U(F)$. The extension of Theorem 0 which is true is

Theorem $0^{\prime}$. If $T$ is coadjoint (order 2$)$, if $T$ bas a cyclic set $\left\{\psi_{0}, \cdots, \psi_{k}\right\}$, and if the spectrum of $T$ is $[0,1]$, then the bilinear form $(F, G)$ can be written as 


$$
(F, G)=\sum_{i, j=0}^{1} \int_{0}^{1} d\left\langle\mu_{i j} F^{(i)}, G^{(j)}\right\rangle
$$

where the $\mu_{i j}$ are $k \times k$ matrices of finite Borel measures on $[0,1]$ and $\mu_{01}=\mu_{10}^{*}$ and $F^{(i)}$ denotes $d^{i} F / d x^{i}$.

Proof. The technique used to prove the scalar case of this representation generalizes in a straightforward way to vector valued testing functions and one can get

$$
(F, G)=\sum_{i, j=0}^{2} \int_{0}^{1} d\left\langle\mu_{i j} F^{(i)}, G^{(j)}\right\rangle .
$$

This was mentioned previously in $\left[\mathrm{H}_{1}\right]$. The problem is to reduce the order of summation from 2 to 1 . The following proposition says that this can be done.

Proposition 2.3. If

REP

$$
(F, G)=\sum_{i=0 ; j=0}^{2} \int_{0}^{1} d\left\langle\mu_{i j} F^{(i)}, G^{(j)}\right\rangle
$$

is a nonnegative bermitian bilinear form in $C^{2}\left[0,1, \mathrm{C}^{n}\right]$ and $\left(d^{3} / d s^{3}\right)\left(e^{i s x} F(x), e^{i s x} G(x)\right)$ $=0$, then $($,$) can be written as in REP but with the sum being taken up to 1$, and witb $\mu_{01}=\mu_{10}^{*}$.

Proof. Let $a$ be a vector in $\mathbf{C}^{n}$ and let $f$ and $g \in C^{2}[0,1]$. The bilinear form $(,)_{a}$ defined by

$$
(f, g)_{a}=\sum_{i=0 ; j=0}^{2} \int_{0}^{1} f \bar{g} d\left\langle a, \mu_{i j} a\right\rangle
$$

satisfies the hypothesis of Theorem 0 . The first few arguments presented in the proof of Theorem 0 imply that $\left\langle a ; \mu_{22} a\right\rangle$ is zero, that $\left\langle a, \mu_{21} a\right\rangle$ and $\left\langle a, \mu_{12} a\right\rangle$ are absolutely continuous with respect to Lebesgue measure and that they have derivatives which are measures. The following lemma implies that each entry of the matrices $\mu_{21}$ and $\mu_{12}$ is differentiable with respect to Lebesgue measure; thus we can eliminate $\mu_{12}$ and $\mu_{21}$ from REP through integration by parts.

Lemma 2.4. Let $\mu$ be a matrix valued measure on $[0,1]$. If $d\langle a, \mu a\rangle=$ $k_{a}(x) d x$ for a function $k_{a}$ of bounded variation, then each entry of $\mu$ is a measure which is actually a function of bounded variation.

Proof. Let $y$ be a complex number of modulus 1 . The polarization identity says

$$
2 \operatorname{Re} \gamma d\langle a, \mu b\rangle=d\langle a+\gamma b, \mu[a+\gamma b]\rangle-d\langle a-\gamma b, \mu[a-\gamma b]\rangle .
$$

Thus $d\left\langle e_{i}, \mu e_{j}\right\rangle=k_{i j}(x) d x$ where $k_{i j}$ is of bounded variation and $e_{i}=$ 
$(0, \ldots, 0,1,0, \ldots, 0)$ with 1 in the $i$ th place. The lemma is proved.

Now we turn our attention to eliminating $\mu_{02}$ and $\mu_{20}$ from REP. Step 1 of Proposition 2.1 demonstrates that $\left\langle\mu_{02} a, a\right\rangle-\left\langle\mu_{20} a, a\right\rangle$ is of bounded variation for each $a$. Thus Lemma 2.4 allows integration by parts to be used in order to put $\mu_{20}=\mu_{02}$ in REP. The argument in Step 2 of Proposition 2.1 obviously applies in this case as well as the scalar case and implies that we may take $\mu_{00}, \mu_{11}, \mu_{02}$ to be hermitian matrix valued measures and that we may take $\mu_{01}=\mu_{10}^{*}$ in REP. Finally, Steps 3 and 4 of Proposition 2.1 imply that $\left\langle\mu_{02} a, a\right\rangle$ is of bounded variation for each $a$. Thus Lemma 2.4 allows integration by parts to be used to eliminate $\mu_{02}$ from REP. Theorem $0^{\prime}$ is proved.

3. Coadjoint operators as sub-Jordan operators. In this section we use the assumption that $T$ has absolutely continuous spectrum in the $A_{2}$ norm and that

$$
\operatorname{Ml} l(\lambda) \geq\left[A_{2} d E_{\lambda} \psi_{0}, \psi_{0}\right] \geq m l(\lambda) .
$$

This assumption when translated into terms of the representation for $(f, g)$ says precisely that

$$
(f, g)=\int_{0}^{1} f \bar{g} d \mu_{00}+\int_{0}^{1} f \bar{g}^{\prime} d \mu_{01}+\int_{0}^{1} f^{\prime} \bar{g} d \bar{\mu}_{01}+\int_{0}^{1} P f^{\prime} \bar{g}^{\prime} d x
$$

where $M \geq P(x) \geq m>0$. The next few pages are devoted to proving consequences of our assumptions on $P$.

Lemma 3.1. $\mu_{01}$ is absolutely continuous with respect to Lebesgue meas ure.

Proof. Suppose not. Suppose that the positive part $\nu$ of $\mu_{01}$ is not 0 on a set $S$ and that $l(S)=0$. Define $S_{N}, S_{N}^{\prime}, S_{N}^{\prime \prime}$ etc. as in Proposition 2.1, Step 3, and set $b_{N}(x)=a_{N} \int_{0}^{x} b_{N}(t) d t+\gamma$. The estimate $\left|b_{N}(x)\right| \leq\left|a_{N}\right| l\left(s_{N}\right)+|\gamma|$ holds for all $x$ in $[0,1]$. Now use equation $(3.1)$ to get

$$
\begin{aligned}
\left(b_{N}, b_{N}\right)= & \int_{0}^{1}\left|b_{N}\right|^{2} d \mu_{00}+\bar{\alpha}_{N} \int_{0}^{1} b_{N} \bar{b}_{N} d \mu_{01}+\alpha_{N} \int_{0}^{1} b_{N} b_{N} d \bar{\mu}_{01} \\
& +\left|a_{N}\right|^{2} \int_{0}^{1}\left|b_{N}\right|^{2} P d x .
\end{aligned}
$$

Take $\alpha_{N}=\left(1 / l\left(S_{N}\right)\right)^{1 / 2}$. Then

$$
\varlimsup\left|\alpha_{N}\right|^{2} \int_{0}^{1}\left|b_{N}\right|^{2} P d x \leq \sup _{[0,1]} P
$$

and

$$
\int_{0}^{1}\left|b_{N}\right|^{2} d \mu_{00} \leq\left|\mu_{00}\right|[0,1](1+|\gamma|)^{2} .
$$

Set $k_{N}=b_{N}-\gamma$. The estimates above and the estimates performed in Proposition 2.1 , Step 3 , when applied to (3.2) yield 
from which we get

$$
\begin{aligned}
0 \leq & M_{\gamma}+a_{N} \int_{0}^{1} k_{N} b_{N} d \mu_{01}+a_{N} \int_{0}^{1} k_{N} b_{N} d \bar{\mu}_{01} \\
& +a_{N} \gamma \int_{0}^{1} b_{N} d \mu_{01}+a_{N} \bar{\gamma} \int_{0}^{1} b_{N} d \bar{\mu}_{01}
\end{aligned}
$$

$$
-M_{\gamma} \leq \alpha_{N}(\gamma+1) \nu\left(C_{N}\right)+\alpha_{N}(\gamma+1) \nu\left(C_{N}\right),
$$

for all $\gamma$ and $N$. This is not possible, since we could take $\gamma=-2$ and since $a_{N} \rightarrow \infty$ while $\nu\left(C_{N}\right) \rightarrow \nu(C)>0$. Thus the positive part of $\mu_{01}$ is absolutely continuous with respect to Lebesgue measure. The negative part is handled similarly.

Now we show that the imaginary part of $\mu_{01}$ is absolutely continuous. Let $b$ be a real function and consider

$$
\left(e^{i b}, e^{i b}\right)=\int_{0}^{1} d \mu_{00}+i \int_{0}^{1} b^{\prime} d\left[\mu_{01}-\bar{\mu}_{10}\right]+\int_{0}^{1} P b^{\prime 2} d x .
$$

If one chooses $b_{N}$ as they were chosen above, then it is easy to get a contradiction to the fact that $0 \leq\left(e^{i b_{N}}, e^{i b_{N}}\right)$. Henceforth, we shall write $d \mu_{01}=r(x) d x$.

Now we are going to prove that $r$ is in $L^{2}$. In order to obtain this it suffices to show that for all $f \in C[0,1]$

$$
\int_{0}^{1}|f r| d x \leq K\left[\int_{0}^{1}|f|^{2} P(x) d x\right]^{1 / 2} .
$$

Suppose that this is not the case. There is a sequence $f_{n}$ of $C[0,1]$ functions for which $\int_{0}^{1}\left|f_{n}\right|^{2} P d x \leq 1$ but $\int_{0}^{1}\left|f_{n} r\right| d x \rightarrow \infty$. Define $b_{n}$ by

$$
b_{n}(x)=\int_{0}^{x} e^{i b(s)} f_{n}(s) d s+\gamma=k_{n}(x)+\gamma
$$

where $e^{i b(s)}|r(s)|=r(s)$. Then

$$
0 \leq\left(b_{n}, b_{n}\right) \leq M_{\gamma}+\int_{0}^{1} b_{n}\left|f_{n}(s) r(s)\right| d s+\int_{0}^{1}\left|f_{n}(s) r(s)\right| \bar{b}_{n} d s
$$

with $M_{\gamma}$ a constant which depends only on $\gamma$. From Schwartz's inequality we have the estimate

$$
\begin{aligned}
\left|\int_{0}^{1} k_{n}\right| f_{n} r|d s| & \leq\left\{\sup _{[0,1]}\left|k_{n}\right|\right\} \int_{0}^{1}\left|f_{n} r\right| d s \\
& \leq\left\{\int_{0}^{1} \frac{1}{P}\left|e^{i b}\right|^{2} d s\right\}^{1 / 2}\left\{\int_{0}^{1}\left|f_{n}\right|^{2} P d s\right\}^{1 / 2} \int_{0}^{1}\left|f_{n} r\right| d s \\
& \leq M \int_{0}^{1}\left|f_{n} r\right| d s .
\end{aligned}
$$

Therefore, we get from (3.4) that $0 \leq M_{\gamma}+2(\gamma+M) \int_{0}^{1}\left|f_{n} r\right| d s$ which is impossible because when $\gamma<-M$ the right side diverges to $-\infty$ as $n \rightarrow \infty$.

Let $H^{1}(l)$ denote the ordinary Lebesgue measure Sobolev space of weakly 
once differentiable functions with norm $\int_{0}^{1}|f|^{2} d x+\int_{0}^{1}\left|f^{\prime}\right|^{2} d x$. The $H^{1}(l)$ norm dominates (, ) because inequality (3.3) along with the classical Sobolev inequalities (see [F]) imply that $\left|\int_{0}^{1} f \vec{f} r d x\right|+\left|\int_{0}^{1} f^{\prime} \bar{f} \bar{r} d x\right|$ is dominated by the $H^{1}(l)$ norm and $\int_{0}^{1}|f|^{2} d \mu_{00}$ is clearly dominated by the $H^{1}(l)$ norm. Thus $H^{1}(l)$ as a set is contained in $H(\mu)$.

The original bilinear form (, ) can be simplified by changing cyclic vectors for $T$. The cyclic vector with which the representation was originally constructed corresponds to the function 1 and the set of all cyclic vectors for $T$ corresponds to the set of all invertible functions in $H^{1}(l)$ as we shall soon see. If we represent $T$ with the cyclic vector which corresponds to the function $c$, then the resulting bilinear form $(f, g)_{c}$ is equal to $(f c, g c)$. Thus we can calculate that $(f, g)_{c}$ has the form

$$
\begin{aligned}
(f, g)_{c}= & \int_{0}^{1} f \bar{g}\left[|c|^{2} d \mu_{00}+c^{\prime} \bar{c} r d x+c^{\prime} \bar{c} \bar{r} d x\right] \\
& +\int_{0}^{1} f^{\prime} \bar{g}\left[|c|^{2} r d x+c^{\prime} P d x\right] \\
& +\int_{0}^{1} f \bar{g}^{\prime}\left[|c|^{2} \bar{r} d x+c^{\prime} \bar{c} P d x\right]+\int_{0}^{1} f^{\prime} \bar{g}^{\prime}|c|^{2} P d x
\end{aligned}
$$

This reduces to

$$
(f, g)_{c}=\int_{0}^{1} f \bar{g} d \dot{\mu}_{00}+\int_{0}^{1} f^{\prime} \bar{g}^{\prime} \dot{P} d x
$$

if we take $r c+P c^{\prime}=0$ and choose $\dot{\mu}_{00}$ and $\dot{P}$ in the obvious way. The function

$$
c(x)=\exp -\int_{0}^{x} r(s) / P(s) d s
$$

which satisfies $r c+P c^{\prime}=0$ is bounded, invertible, and is in $H^{1}(l)$. Thus $c$ is a cyclic vector under multiplication by $x$ in $H(\mu)$. Henceforth we shall write $P$ and $\mu_{00}$ instead of $\dot{P}$ and $\dot{\mu}_{00}$ and we shall take $H(\mu)$ to be the Hilbert space of functions with $(,)_{c}$ norm given by (3.6). It is clear from the simple form of $(,)_{c}$ that it is equivalent to the $H^{1}(l)$ norm.

Remark 3.1. The process of changing cyclic vector is analogous to putting the second order differential equation $p y^{\prime \prime}+r y^{\prime}+q y=0$ into Sturm-Liouville form by multiplying through by $\exp -\int_{0}^{x} r / p$.

Remark 3.2. A vector $c$ in $H^{1}(l)$ and consequently in $H(\mu)$ is cyclic for 'multiplication by $x$ ' if and only if $c$ is bounded and bounded away from zero. Thus the effect on $P(x)$ of a change of cyclic vector for $T$ acting on $H$ is to multiply it by a bounded invertible function (see calculation (3.5)). This shows that the Technical Assumption of Theorem II is invariant under change of cyclic vector.

This concludes our discussion of the basic consequences of the Technical 
Assumption of Theorem II. Now we turn to the proof of Theorem II and begin with some preliminaries.

Proposition 3.1. If $b$ is (, ) orthogonal to $H^{1}(l)_{0}=\left\{f \in H^{1}(l): f(1)=0\right\}$ and if $b(0)=0$, then $b$ is identically zero.

Remark. This is a generalization of the familiar uniqueness theorem for Sturm-Liouville operators.

Proof. Let $S_{n x_{0}}$ denote the approximate Heaviside function defined by

$$
S_{n x_{0}}(x)= \begin{cases}1 & \text { if } x \leq x_{0}-1 / n, \\ (\pi / 2)\left(x-x_{0}\right)+1 / 2 & \text { if } x_{0}-1 / n \leq x \leq x_{0}+1 / n, \\ 0 & \text { if } x_{0}+1 / n \leq x .\end{cases}
$$

For each Lebesgue point $x$ of $P b^{\prime}$ we have, after taking the limit as $n \rightarrow \infty$ in representation (3.6) for $\left(b, S_{n x_{0}}\right)=0$,

$$
\int_{0}^{x} b d \mu_{00}=-P(x) b^{\prime}(x)
$$

Thus (since $b(0)=0$ )

$$
h(x)=-\int_{0}^{x} \frac{1}{P} \int_{0}^{s} b d \mu_{00} .
$$

Now we invoke a standard argument. Equation (3.7) implies $|h(x)| \leq\left\{(1 / \alpha) \int_{0}^{1} d\left|\mu_{00}\right|\right\} \mathbf{H}(x) x$ where $H(x)=\sup _{[0, x]}|h(s)|$ and $P(x) \geq a>0$. Let $X$ be a number such that $\rho=(1 / \alpha) \int_{0}^{1}\left|\mu_{00}\right| d x<1 / X$, and let $m$ be a number $\leq X$ such that $|b(m)|=\mathbf{H}(X)$. Then $\mathbf{H}(x)=|b(m)| \leq \rho m \mathbf{H}(m)<\mathbf{H}(X)$ and we may conclude that $\mathbf{H}(X)=0$. Since $b$ is in $H^{1}(l)$ it is continuous and there must be some largest point $x_{1}$ in $[0,1]$ such that $b$ is identically zero to the left of it. The above argument applied at $x_{1}$ rather than 0 gives a contradiction. This completes the proof of Proposition 3.1.

In what follows we shall employ a bilinear form $(,)_{\beta}$ which is a slight modification of $(,)_{c}$. If $\beta$ is a positive number define $(,)_{\beta}$ by

$$
(f, g)_{\beta}=(f, g)_{c}-\beta \int_{0}^{1} f \bar{g} P d x .
$$

The original assumption POL 2 along with the construction of the representation (3.6) implies that $\exists$ a form $M(f, f)$ such that

$$
\begin{aligned}
O(s) M(f, f)+s^{2} \int_{0}^{1}|f|^{2} P d x= & \left\|\Phi(f) \psi_{0}\right\|^{2}+s\left[A_{1} \Phi(f) \psi_{0}, \Phi(f) \psi_{0}\right] \\
& +s^{2}\left[A_{2} \Phi(f) \psi_{0}, \Phi(f) \psi_{0}\right]
\end{aligned}
$$


where $O(s) / s^{2} \rightarrow 0$ as $s \rightarrow \infty$. Now divide through by $s^{2}$, let $s \rightarrow \infty$, and use the fact that $\left[A_{2} \Phi(f) \psi_{0}, \Phi(f) \psi_{0}\right] \leq\left\|A_{2}\right\|\left[\Phi(f) \psi_{0}, \Phi(f) \psi_{0}\right]=\left\|A_{2}\right\|(f, f)$ to obtain $\int_{0}^{1}|f|^{2} P d x \leq\left\|A_{2}\right\|(f, f)_{c}$. Henceforth, we shall always take $\beta$ to be less than $1 /\left\|A_{2}\right\|$. This implies that $(,)_{\beta}$ is positive definite, and consequently the basic facts about $(,)_{c}$ also hold for $(,)_{\beta}$. In particular $\sqrt{(,)_{\beta}}$ is equivalent to the $H^{1}(l)$ norm, and Proposition 3.1 holds for $(,)_{\beta}$.

Let $b \in H^{1}(l)$ denote the real valued function with normalization $b(1)=1$ which is $(,)_{\beta}$ orthogonal to $H^{1}[l]_{0}=\left\{f \in H^{1}(l): f(1)=0\right\}$. Such a function exists and is uniquely determined by this condition. Any function $k$ with $k(1)=1$ can be written $k=b+f$ where $f \in H^{1}[l]_{0}$ and one can write $(k, k)_{\beta}=(b, b)_{\beta}+$ $(f, f)_{\beta}$. Since $(f, f)_{\beta} \geq 0, k$ is a minimum of $(k, k)_{\beta}$ subject to the side condition $k(1)=1$ if and only if $k \equiv b$.

Suppose that $b$ has a zero at a point $a$ in $[0,1]$. Split $b$ into two parts, $b=$ $b_{0}+b_{1}$, defined by

$$
b_{0}(x)=\left\{\begin{array}{ll}
b(x) & \text { if } x \leq a, \\
0 & \text { if } x \geq a ;
\end{array} \quad b_{1}(x)= \begin{cases}0 & \text { if } x \leq a, \\
b(x) & \text { if } x \geq a .\end{cases}\right.
$$

Since $b \in H^{1}[l]$, it is straightforward to check that both $b_{0}$ and $b_{1}$ are in $H^{1}[l]$. Furthermore, $\left(b_{0}, b_{1}\right)_{\beta}=0$ because $b_{0}$ and $b_{1}$ have disjoint support. Thus

$$
(b, b)_{\beta}=\left(b_{0}, b_{0}\right)_{\beta}+\left(b_{1}, b_{1}\right)_{\beta} \text {, }
$$

but the minimum property of $b$ implies that $\left(b_{0}, b_{0}\right)_{\beta}=0$. Thus $b=b_{1}$. However, $b_{1}(0)=0$ and so Proposition 3.1 implies that $b_{1} \equiv 0$.

Consider the bilinear form

$$
p(f, g)=(f, g)_{c}-(f \bar{g} / h, b)_{\beta}+f(1) \bar{g}(1)(1, b)_{\beta}
$$

where $b$ is again the function $(,)_{\beta}$ orthogonal to $H^{1}[l]_{0}$ with $b(1)=1$. We have

$$
\begin{aligned}
p(f, g)= & \int_{0}^{1} f \bar{g}\left(\frac{b^{\prime 2}}{b^{2}}+\beta\right) P d x-\int_{0}^{1} f \bar{g}^{\prime} \frac{b^{\prime}}{b} P d x-\int_{0}^{1} f^{\prime} \bar{g} \frac{b^{\prime}}{b} P d x \\
& +\int_{0}^{1} f^{\prime} \bar{g}^{\prime} P d x+f(1) \bar{g}(1)(1, b)_{\beta} .
\end{aligned}
$$

The definition of $b$ implies that $(f, b)_{\beta}=f(1)(1, b)_{\beta}$ and so $p(f, g)$ is actually $(f, g)_{c}$. Also, $0<(b, b)_{\beta}=b(1)(1, b)_{\beta}$ which gives us that $(1, b)_{\beta}$ is positive. At last we have reached our goal and represented $(f, g)_{c}$ using a matrix of measures 


$$
\left(\begin{array}{ll}
\left(b^{\prime} / b\right)+\beta & -b^{\prime} / b \\
-b^{\prime} / h & 1
\end{array}\right) P+\left(\begin{array}{ll}
\operatorname{dirac} \delta \text { at } 1 & 0 \\
0 & 0
\end{array}\right)(1, b)_{\beta}
$$

which (at $x \neq 1$ ) has determinant $\beta P(x)$ and consequently is positive definite. This completes the proof of Theorem II.

4. Extensions and other directions. Many generalizations of the results of this paper seem plausible. The goal of this paper has been to work through a simple case completely and generality has not been pursued. In this section we indicate several directions in which one might extend results of this paper and we also give some results concerning the geometric properties of coadjoint operators.

Most of the techniques from analysis used in proving Theorem $I$ have very well-known generalizations which probably lead to generalizations of Theorem II. For example, Theorem $0^{\prime}$ extends the Sobolev space representation theorem to coadjoint operators $T$ with finite multiplicity by using vector rather than scalar valued functions. The extension of the conjugate point theorem to the vector valued case is well known (see Chapter 5, $\$ 29$ [G-F]). It should be possible to combine these two methods to extend Theorem II to the finite multiplicity case. If $\psi_{1}, \ldots$, $\psi_{n}$ is a "cyclic set" for $T$, then the Technical Assumption that the $n \times n$ matrix inequality

$$
m l(s) I \leq\left\langle\left[A_{2} E(s) \psi_{i}, \psi_{j}\right]\right\rangle \leq M l(s) I
$$

holds for all Borel sets $S$ in $\sigma(T)=[a, b]$ is the natural analog of the Technical Assumption for one cyclic vector.

We define a Pontryagin $\Pi_{k}$ space to be the sequence space $l^{2}[-k, \infty]$ with the "J-inner product"

$$
\langle\underline{x}, \underline{y}\rangle=\sum_{l=0}^{\infty} x_{l} \bar{y}_{l}-\sum_{l=-k}^{-1} x_{l} \bar{y}_{l} .
$$

A bibliography of the subject appears in $[K]$. If $T$ is an $l^{2}[-K, \infty]$ bounded operator, its adjoint with respect to the "J-inner product" is denoted by $T^{\circ}$. The notions of $J$-Jordan, $J$-sub-Jordan, and J-coadjoint operators are obtained if one substitutes ${ }^{\circ}$ for ${ }^{*}$ in our original definitions. Steps $(1.1)$ to $(1.7)$ in the proof of Theorem I naturally go through for the $\Pi_{k}$ case, since they are entirely algebraic. However, the rest of the proof poses problems. Theorem II for $J$-coadjoint operators probably reduces to the fact that the number of negative eigenvalues of a Sturm-Liouville operator $L$ is the same as the number of zeroes of a solution to $L y=0$. 
Two commuting coadjoint operators can be represented as multiplication by $x$ and $y$, respectively, on a "Sobolev space" over $R^{2}$ (see $\left[\mathrm{H}_{1}\right]$ ). Possibly the study of two commuting coadjoint operators is connected with conjugate curve theory in the plane.

There are several ways to extend Theorem 0 . The type of argument used in proving Theorem REP can be used to show that any operator $T$ with $C^{n}\left(R^{1}\right)$ functional calculus (see Chapter 5 of $[C-F]$ ) has a representation as "multiplication by $x$ " on a space of functions with inner product

$$
\begin{aligned}
(f, g) & =\left[\phi(f) \psi_{0}, \phi(g) \psi_{0}\right]=b(f(x) \bar{g}(y)) \\
& =\sum_{l, j=0}^{M} \iint_{c-\epsilon}^{d+\epsilon} K_{l j}(x, y) f^{(l)}(x) g^{(j)}(y) d x d y .
\end{aligned}
$$

Here $\sigma(T)=[c, d]$. Various hypotheses on $T$ impose conditions on the distribution $b$ and consequently on the kernels $K_{i j}$ in the representation for $b$. If $T$ satisfies a polynomial condition $P\left(T^{*}, T\right)=0$ where $P(x, y)=\Sigma^{M} a_{n m} x^{n} y^{m}$ and $P\left(T^{*}, T\right)=\Sigma^{M} a_{n m} T^{* n} T^{m}$, then $b(p(x, y) f(x) \bar{g}(y))=0$ for all $f_{,} g \in \mathcal{S}(R)$, and consequently the distribution $b$ has support on the variety of zeroes of $p(x, y)$. The coadjoint operators constitute the special case where $p(x, y)=(x-y)^{n}$. Any restriction which one puts on the function $R(s)=e^{-i s T^{*}} e^{i s T}$ translates into a restriction on the Fourier transforms of $b$ in the direction perpendicular to the diagonal $\{(x, x)\}$, since

$$
\left[e^{i s T} \phi(f) \psi_{0}, e^{i s T} \phi(g) \psi_{0}\right]=b\left(e^{i s[x-y]} f(x) \bar{g}(y)\right) \text {. }
$$

A condition more general than the coadjointness condition on an operator $T$ is that $R(s)$ has an asymptotic expansion

$$
e^{-i s T^{*}} e^{i s T} \sim \sum_{l=-\infty}^{n} s^{l} A_{l}
$$

Such information on $R(s)$ gives one information on the behavior of $b$ near the diagonal $\{(x, x)\}$.

$A$ rather obvious undertaking would be to extend Theorems I and II to cover coadjoint operators of order higher than 2. Colojara and Foias, discuss in [C-F] the notion of two operators being quasi-nilpotent equivalent. The author prefers an equivalent formulation of the definition which says that the operators $A$ and $B$ are quasi-nilpotent equivalent if and only if the functions $\left\|e^{-z A} e^{z B}\right\|$ and $\left\|e^{-z B} e^{z A}\right\|$ of $z$ have exponential order 0 . An advanced version of Theorem I which might be true is-the operator $T$ is a Jordan operator with $N$ quasi-nilpotent if and only if $T$ and $T^{*}$ are quasi-nilpotent equivalent.

We shall call an operator $T$ orthogonally separated if any two invariant sub- 
spaces $\mathcal{X}$ and $Y$ of $T$ with the property that $\sigma\left(\left.T\right|_{X}\right)$ does not intersect $\sigma\left(\left.T\right|_{y}\right)$ are orthogonal. Multiplication by $x$ on a $H(\mu)$ space, and also multiplication by $x+i y$ on a generalized Sobolev space are orthogonally separated operators. The converse is also true.

Proposition 4.1. If $T$ bas a $C^{n}\left(R^{2}\right)$ functional calculus, if $T$ is orthogonally separated, if $T$ bas a cyclic vector $\psi_{0}$, and if the spectrum of $T$ is a simply connected set with smooth boundary, then $T$ is unitarily equivalent to "multiplication by $x$ " on the usual sort of weighted Sobolev space supported over $\sigma(T)$.

Note. Many of the hypotheses can be lifted in the way which we already described.

Idea of proof. The analogous argument to that of Theorem REP says that there is a distribution $b$ such that $\left[\phi(f) \psi_{0}, \phi(g) \psi_{0}\right]=b(f(x, y) \bar{g}(\xi, \zeta))$ for each $f, g \in \mathcal{S}\left(R^{2}\right)$. The assumption that $T$ is orthogonally separated implies that if $f$ and $g$ have disjoint supports, then $b(f \bar{g})=0$. This is equivalent to saying that $b$ has support on the diagonal $\{(x, y),(x, y)\}$. Now one can continue using the argument behind Theorem REP and get the two dimensional analog of equation (2.1). The main difference is that in the two dimensional case some power is required to restrict the doma in of integration to $\sigma(T)$.

The connection between orthogonally separated operators and coadjoint operators is given by

Proposition 4.2. The operator $T$ bas $C^{n}\left(R^{1}\right)$ functional calculus and is orthogonally separated if and only if $T$ is coadjoint.

Proof. Suppose that $T$ is orthogonally separated and has a $C^{n}\left(R^{1}\right)$ functional calculus. Let $\psi$ denote a vector in $H$ and let $\mathbb{N}(\psi)=$ closure of $\{V \in H: V=$ $\phi(f) \psi$ for some $\phi(f)$ given by $(2.0)\}$. By Theorem REP the operator $T$ restricted to $\Pi(\psi)$ is unitarily equivalent to "multiplication by $x$ " on a $H(\mu)$ space of order $\leq 2 n$. Consequently $\left.T\right|_{\pi(\psi)}$ is coadjoint (of order $\leq 2 n$ ). Thus we have shown that for any $\psi$ in $H$

$$
\left(d^{2 n} / d s^{2 n}\right)\left[e^{i s T} \psi, e^{i s T} \psi\right]=0
$$

and so $T$ is coadjoint.

Suppose that $T$ is coadjoint (order $k$ ). Then the estimate $\left\|e^{i s T}\right\| \leq O\left(s^{k}\right)$ holds and Theorem V $4.5[\mathrm{C}-\mathrm{F}]$ says that $T$ has a $C^{n}\left(R^{1}\right)$ functional calculus. Suppose that $X_{1}$ and $X_{2}$ are invariant subspaces of $T$ and that $\sigma\left(T \mid x_{1}\right) \cap$ $\sigma\left(\left.T\right|_{x_{2}}\right)=\varnothing$. Let $\psi_{1}$ be a vector in $X_{1}$ and $\psi_{2} \in X_{2}$ and let $\mathbb{M}\left(\psi_{1}, \psi_{2}\right)=$ closure of $\left\{V \in H: V=\phi\left(f_{1}\right) \psi_{1}+\phi\left(f_{2}\right) \psi_{2} \text { for some } \phi\left(f_{1}\right) \text { and } \phi\left(f_{2}\right)\right\}_{\text {. A A version }}$ of Theorem REP for a finite cyclic set $\left(\right.$ see $\left[\mathrm{H}_{1}\right]$ or Theorem $\left.0^{\prime}\right)$ yields 


$$
\begin{aligned}
{\left[\phi\left(f_{1}\right) \psi_{1}+\phi\left(f_{2}\right)\right.} & \left.\psi_{2}, \phi\left(g_{1}\right) \psi_{1}+\phi\left(g_{2}\right) \psi_{2}\right] \\
& =\sum_{i, j=0}^{M} \sum_{a, \beta=1}^{2} \int_{-\infty}^{\infty} f_{a}^{(i)} \bar{g}_{\beta}^{(j)} d \mu_{i j a \beta}
\end{aligned}
$$

for some Borel measures $\mu_{i j a \beta}$. Consequently, if $f$ and $g$ have disjoint support then $\left[\phi(f) \psi_{1}, \phi(g) \psi_{2}\right]=0$. Since $\left.T\right|_{\pi\left(\psi_{j}\right)}$ is coadjoint and the functional calculus homomorphism for it is just $\left.\phi(f)\right|_{\Re\left(\psi_{j}\right)}$, Lemma I in $\left[\mathrm{H}_{1}\right]$ implies that $\phi(f) \psi_{j}$ $=\psi_{j}$ if $f$ is identically one on $\sigma\left(\left.T\right|_{\pi\left(\psi_{j}\right)}\right.$. Since there exist two functions $f_{1}$ and $f_{2}$ in $\mathcal{S}$ with dis joint support which are 1 on $\sigma\left(\left.T\right|_{\Re\left(\psi_{1}\right)}\right)$ and $\sigma\left(\left.T\right|_{\pi\left(\psi_{2}\right)}\right)$ respectively, we may conclude that $\left[\psi_{1}, \psi_{2}\right]=\left[\phi\left(f_{1}\right) \psi_{1}, \phi\left(f_{2}\right) \psi_{2}\right]=0$. Thus we see that $X_{1}$ and $X_{2}$ are orthogonal and we have proved that $T$ is orthogonally separated.

One consequence of Proposition 4.2 is that Theorem I becomes

Theorem 4.3. The operators $T$ and $T^{*}$ are orthogonally separated and satis. fy the estimate $\left\|e^{-i s T}\right\| \leq O(|s|)$ if and only if $T$ is a Jordan operator with nilpotent part of order 2.

The author suspects that the most practical way of generalizing Theorem I to higher orders is with a proof which uses analysis and the geometric notion of orthogonally separated operators. It also seems likely that the operators of the form $A+N$ where $A$ is normal, $A N=N A$ and $N$ is nilpotent are those which are orthogonally separated, have orthogonally separated adjoints and which have $C^{n}\left(R^{2}\right)$ functional calculus. We conclude this line of discussion by suggesting that it might be interesting to study orthogonally decomposable operators, namely, orthogonally separated operators which are decomposable in the sense of [C-F, Chapter 2].

This paper purports to study the two simplest types of operators with real spectrum. We conclude by suggesting a candidate for the "third simplest" type of operator with real spectrum. In [S] Sarason calls a subspace $S$ semi-invariant for an operator $M$ if it is the orthogonal difference $S=N_{1} \Theta N_{2}$ of two invariant subspaces $N_{1}$ and $N_{2}$ where $N_{2}$ is contained in $N_{1}$. We shall say that an operator $T$ on $H$ is the compression of an operator $M$ on $\mathcal{H}$ if $T=\left.P_{H} M\right|_{H}$ where $H$ is a semi-invariant subspace for $M$ and $P_{H}$ is the orthogonal projection of $\mathcal{H}$ onto $H$. The case where $M$ is unitary plays a central role in [L-P] and $[N-F]$, one of the standard operations (although it has never been described in these terms) in the study of operators on a J-space (cf. [Ph, \$4]) amounts to "compressing" a J-unitary operator $M$ to a semi-invariant subspace of $M$; and an abstract study of compressions has been made in $[A]$. We shall call the compression of a Jordan operator a compressed Jordan operator. One can find an example of these operators in 
the abstract study of partial differential equations using $J$-spaces proposed in $[\mathrm{Ph}$. Compressed Jordan operators seem very natural and a study of them should be interesting.

\section{BIBLIOGRAPHY}

[A] W. B. Arveson, Subalgebras of $C^{*}$ algebras. II (to appear).

[C-F] I. Colojara and C. Foias,, Theory of generalized spectral operators, Gordon and Breach, New York, 1968.

[D-S] N. Dunford and J. T. Schwartz, Linear operators. I: General theory, Pure and Appl. Math., vol 7, Interscience, New York, 1958. MR 22 \#8302; II: Spectral theory. Selfadjoint operators in Hilbert space, Interscience, New York, 1963. MR 32 \#6181.

[G-F] I. M. Gel' fand and S. V. Fomin, Calculus of variations, Fizmatgiz, Moscow, 1961; English transl., Prentice-Hall, Englewood Cliffs, N. J., 1963. MR 28 \#3352; \#3353.

[F] A. Friedman, Partial differential equations, Holt, Rinehart and Winston, New York, 1969.

[H $\left.\mathrm{H}_{1}\right] \mathrm{J}$. W. Helton, Operators with a representation as multiplication by $x$ on a Sobolev space, Proc. Internat. Conference on Operator Theory, Hungary, 1970.

$\left[\mathrm{H}_{2}\right]-$ Jordan operators in infinite dimensions and Sturm-Liouville conjugate point theory, Bull. Amer. Math. Soc. 78 (1972), 57-61.

[K] M. G. Krěn, Introduction to the geometry of indefinite J-spaces and to the theory of operators in those spaces, Second Math. Summer School, part 1, "Naukova Dumka", Kiev, 1965, pp. 15-92; English transl., Amer. Math. Soc. Transl. (2) 93 (1970), 103-176. MR 33 \#574; MR 42 \#4.

[L-P] P. D. Lax and R. S. Phillips, Scattering theory, Pure and Appl. Math., vol 26, Academic Press, New York, 1967. MR 36 \#530.

$[\mathrm{Ph}] \mathrm{R}$. S. Phillips, The extension of dual subspaces invariant under an algebra, Proc. Internat. Sympos. Linear Spaces (Jerusalem, 1960), Jerusalem Academic Press, Jerusalem; Pergamon Press, Oxford, 1961, pp. 366-398. MR 24 \#A3512.

[N-F] B. Sz. Nagy and C. Foias,, Analyse harmonique des opérateurs de l'espace de Hilbert, Masson, Paris; Akad. Kiadó, Budapest, 1967; English transl., North-Holland, London, 1970. MR 37 \#778.

[R] H. Royden, Real analysis, Macmillan, New York, 1963. MR 27 \#1540.

[s] D. Sarason, On spectral sets having connected complement, Acta Sci. Math. (Szeged) 26 (1965), 289-299. MR 32 \#6229.

DEPARTMENT OF MATHEMATICS, STATE UNIVERSITY OF NEW YORK AT STONY BROOK, STONY BROOK, NEW YORK 11790 